\title{
Du poulailler au marché
}

Esquisse d'une économie volaillère médiévale (XIII $-\mathrm{XVI}^{\mathrm{e}}$ siècle)

From henhouse to market: Sketch of a medieval poultry economy (13th-16th century)

\section{Mickaël Wilmart}

\section{OpenEdition}

Journals

Édition électronique

URL : http://journals.openedition.org/ethnoecologie/3318

DOI : $10.4000 /$ ethnoecologie.3318

ISSN : 2267-2419

\section{Éditeur}

Laboratoire Eco-anthropologie et Ethnobiologie

\section{Référence électronique}

Mickaël Wilmart, «Du poulailler au marché », Revue d'ethnoécologie [En ligne], 12 | 2017, mis en ligne le 18 décembre 2017, consulté le 10 décembre 2020. URL : http://journals.openedition.org/ ethnoecologie/3318; DOI : https://doi.org/10.4000/ethnoecologie.3318

Ce document a été généré automatiquement le 10 décembre 2020.

\section{(c) (i) (9)}

Revue d'ethnoécologie est mis à disposition selon les termes de la licence Creative Commons Attribution - Pas d'Utilisation Commerciale - Pas de Modification 4.0 International. 


\title{
Du poulailler au marché
}

\author{
Esquisse d'une économie volaillère médiévale (XIII ${ }^{\mathrm{e}}-\mathrm{XVI}^{\mathrm{e}}$ siècle) \\ From henhouse to market: Sketch of a medieval poultry economy (13th-16th \\ century)
}

Mickaël Wilmart

1 Alors que la médecine vétérinaire concentre ses efforts sur les animaux de prestige comme les chevaux ou les faucons (Wilmart 2017a), les soins prodigués aux poules ne sont pas absents des textes médiévaux. Dans son traité d'économie rurale, Pierre de Crescens évoque deux pathologies à surveiller: la pépie et une affection oculaire que l'on doit assimiler à une forme de cataracte (Crescens 1516: f. 112-113). Pour chacune, il décrit l'opération chirurgicale à effectuer sur le volatile. L'intervention sur la pépie semble d'ailleurs courante si l'on en juge au rôle de référence qu'elle joue chez certains auteurs. Ainsi, à la fin $\mathrm{du} \mathrm{xv}^{\mathrm{e}}$ siècle, dans son traité de fauconnerie, au chapitre de la pépie, Guillaume Tardif conseille de l'ôter « comme on fait aux poulles» (Tardif 1492: 48). La mention d'un protocole opératoire sur l'œil, s'il est régulier sur des bêtes plus importantes, est unique pour l'espèce et s'oppose à l'injonction de tuer un pigeon qui souffrirait du même mal (Crescens 1516: f. 116). Assurément, les gallinacés méritent davantage de soins que les colombidés sans doute parce qu'ils s'inscrivent dans un circuit économique plus diversifié dans lequel sont mis en circulation leur viande mais aussi leurs œufs. Le calcul rationnel n'est d'ailleurs pas étranger à l'élevage avicole. Au XIII ${ }^{\mathrm{e}}$ siècle, dans son traité de Husbrandry, Walter de Henley indique qu'idéalement chaque poule doit pondre 115 œufs par an, donner sept poussins dont quatre futures pondeuses et trois qui seront transformés en chapons (Oschinsky $1971: 424$ ).

2 Les quelques exemples tirés de traités cachent toutefois une réalité beaucoup plus aride. En effet, les sources écrites sont, en général, peu prolixes sur l'économie de la basse-cour. Le plus souvent, l'historien devine l'existence d'un élevage domestique de volailles mais ne peut en dire beaucoup plus. Pour en esquisser un portrait plus détaillé, il est alors nécessaire de relire les archives déjà maintes fois commentées, notamment celles qui concernent les prélèvements seigneuriaux, mais pour les relire différemment. On peut alors les confronter avec quelques extraits de comptabilités seigneuriales mentionnant la 
circulation des volailles, des textes de coutumes ou de franchises donnant pour certaines un détail parlant. Il faut aussi avoir recours aux archives judiciaires, notamment dans leur échelon le plus bas, pour en tirer des pratiques autrement invisibles. Enfin, avec le traité rédigé par Prudent Le Choyselat à la fin $\mathrm{du} \mathrm{XVI}^{\mathrm{e}}$ siècle émerge un nouveau modèle d'aviculture qui, s'il est avant tout un jeu de l'esprit de la part de son auteur, éclaire aussi le système économique en vigueur jusque-là, parce qu'il entend à la fois le modifier tout en s'appuyant sur des pratiques et des structures déjà existantes.

Cette confrontation de deux modèles conclura cet article. Avant cela, je me propose d'examiner les prélèvements seigneuriaux et les redevances comme une économie du surplus. Celle-ci n'est alors qu'une partie d'un circuit plus complexe dont mise sur le marché et échanges villageois dessinent la trame.

\section{Prélèvements seigneuriaux et redevances : une économie du surplus?}

$4 \quad$ Les cens et autres redevances payables en poules constituent souvent les seules allusions aux volailles dans les grandes monographies régionales d'histoire rurale. Ce laconisme est essentiellement dû aux sources utilisées (et généralement disponibles) d'origine seigneuriale, donnant plus à voir les revenus tirés de la seigneurie que les pratiques d'élevage. Les conclusions qu'en tirent les historiens médiévistes ne sont pas univoques. Si, pour Guy Fourquin, les mentions de "gélines » dans les cens et autres prélèvements sont le signe d'une "extension considérable » de la "poulaille » parmi les paysans et les seigneurs d'île-de-France (Fourquin 1964: 80)1, Pierre Charbonnier est plus circonspect à ce sujet. Pour lui, à partir du cas auvergnat, « on ne peut pas trop se fonder sur les gélines des redevances » (Charbonnier $1980:$ 185). Même si celles-ci sont omniprésentes, il est difficile d'apprécier l'aviculture à partir de ces mentions. En fait, comme on le verra dans la suite de cet article, il est difficile de ne pas partager le pessimisme de Pierre Charbonnier, prélèvements et redevances n'étant pas à considérer comme un témoignage d'une activité généralisée mais comme une des voies de circulation économique des produits de la basse-cour.

Ces redevances ne sont pas toutes de même nature et s'appliquent selon des logiques fort différentes. Les cens dus en argent ou en nature (et parfois donc en poules) sont associés à des tenures et liés à la terre exploitée par le paysan. Ce type de redevance peut être payé en volailles si le seigneur le décide, mais ce paiement est loin d'être généralisé. Le principal prélèvement qui donne lieu à une intense circulation volaillère peut être regroupé sous le terme générique de fouage, même si les usages locaux lui donnent parfois un autre nom. C'est une redevance payée par feu, c'est-à-dire par maison ou famille. Elle conclut une reconnaissance personnelle entre un seigneur et son sujet. Le paiement de cette reconnaissance par la livraison d'une poule est quasiment généralisé dans le sud du royaume. On la rencontre également dans le nord de la France. Dans les comptes du temporel de l'évêque de Meaux de 1425, on trouve, d'une part, des poules et des chapons dus en cens (mais qui sont alors convertis en deniers) et, d'autre part, des poussins dus en fouage au village de Varredes et des gélines au village de Germigny (Parfouru 1900:6). En Gascogne, la plupart des coutumes accordées aux communautés villageoises comportent la mention d'une redevance en poule par feu. À Labejean, en 1313, chaque chef de famille est ainsi tenu de payer à la Toussaint une paire de poules, 
quel que soit par ailleurs le nombre de maisons ou de terres qu'il possède ou loue dans le territoire (Bladé 1864: 49). Le texte précise également qu'en cas de division des biens entre plusieurs frères, chacun sera tenu de payer la redevance. Cette dernière disposition se retrouve encore en 1553 à Aguin (Bladé 1864 : 92-93). Le seigneur peut aussi exiger de la volaille ainsi prélevée une certaine qualité. Par exemple, en 1283, à Bivès, la géline doit être en état de faire des œufs (Bladé 1864 : 22). Rachetant parfois explicitement la corvée, comme dans le même village, la redevance est due de façon égale par tous les feux sans rapport avec la fortune ou la position sociale. Ainsi, les coutumes d'Endoufielle, accordées en 1261, fixent une redevance d'une poule ou de deux poulets (unam gallinam vel duos gallinatos pro gallina) que l'imposé soit laboureur travaillant avec deux ou plusieurs animaux, laboureur avec un seul animal, brassier, étranger ou femme célibataire (Laporte 1911, 9-10). Devant de tels textes normatifs, on doit bien sûr se poser la question de la généralisation de l'élevage de poules, d'autant que les documents eux-mêmes le laissent parfois penser. En Languedoc, à La Salvetat-sur-Agout, l'évêque de Saint-Pons-deThomières a droit de prendre une poule sur chaque maison, ses officiers devant aller la chercher « alz houstalz d'un chacun » (Sahuc 1895 : 108-109). Il faut toutefois se garder de conclure trop vite. Certaines chartes, comme à Bivès, précisent que tout le monde ne doit pas ce type de redevances. Progressivement, comme on le voit au final à Meaux au $\mathrm{Xv}^{\mathrm{e}}$ siècle, certains prélèvements sont convertis en $\operatorname{argent}^{2}$, et ce, dès le XIII ${ }^{\mathrm{e}}$ siècle. En Languedoc toujours, à Olargues, le premier article des franchises accordées en 1289 porte sur cette question. S'ils le souhaitent, les habitants peuvent transformer le cens dû en géline en la somme de sept deniers. En ce qui concerne la poule réclamée pour chaque feu, seul le seigneur peut exiger la conversion en deniers, au cas où il juge insuffisant (ou impossible ?) le paiement en nature (Sahuc 1903 : 313). On entrevoit, à travers l'exemple d'Olargues, les difficultés rencontrées dans le prélèvement de volailles, signe de l'absence d'une généralisation. De fait, au cours des XIV et $\mathrm{XV}^{\mathrm{e}}$ siècles, le nombre de redevances en poules tend dans plusieurs endroits à se réduire. Ainsi, à la fin $\mathrm{du} \mathrm{XIV}^{\mathrm{e}}$ siècle, en Lauragais, le revenu des gélines est encore listé dans les aveux et dénombrements même si celui-ci est loin d'être systématique (Marandet 2006 : 318-321). Entre la fin du XIV et la fin du Xv siècle, les redevances en poules ont tendance à baisser, comme le montre l'exemple de la seigneurie de Fendeille entre 1383 et 1463 (Marandet 2006: 321). Dans la seconde moitié $\mathrm{du} \mathrm{Xv}^{\mathrm{e}}$ siècle, on peut faire la même constatation dans le Vivarais. À Vallon-Pont d'Arc, en 1464, sur les 1114 cens recensés dans les estimes, seuls 17 sont à payer en poules, soit moins de $2 \%$ (Valladier-Chante 1993: 65). Toutefois, cette baisse n'est pas partout la règle. En 1474, l'inventaire des revenus de l'évêque de Die mentionne encore un peu plus de cinq cents poules prélevées en différents endroits (Fillet 1890).

6 Au-delà des archives seigneuriales, les minutes de notaires pourraient éventuellement nous donner des indications plus précises, mais, comme le note Marie-Claude Marandet pour le Lauragais, les mentions de volailles sont plutôt rares. On ne les trouve jamais citées dans les contrats de vente d'animaux, le coût de la vente étant sans doute trop faible pour entreprendre la rédaction d'un acte devant notaire. En revanche, on les rencontre régulièrement parmi les redevances listées dans les baux de fermage ou de métayage (Marandet 2006 : 174). Ces baux contiennent en effet parfois des redevances qui semblent être les héritières des anciens prélèvements seigneuriaux et ils obligent, dans un certain nombre de cas, à la fourniture régulière de volailles. Ainsi, en 1460 , à Ayguesvives, un fermier de Raymond de Pechbusque, qui habite Toulouse, doit lui fournir quatre paires de coqs à la Pentecôte, quatre paires de poules à la Saint-Thomas et 
cinquante œufs à Pâques. Quant au métayer de Guilhem-Azémar de Roqueville, dans le village voisin, il doit à son propriétaire cinq paires de poules à Noël et cinq paires de coqs à la Saint-Jean-Baptiste (1408). Les riches marchands se font aussi livrer des volailles : en 1407 et 1408, Aymeric de Miralhis impose à ses métayers la livraison de six paires de coqs à la Saint-Jean-Baptiste et quatre paires de poules à Noël (Marandet 2006:362). La généralisation de la pratique du métayage à mi-fruit au cours $\mathrm{du} \mathrm{XVI}$ siècle, beaucoup plus favorable au preneur, amplifie le phénomène de circulation de volailles entre l'exploitation et le propriétaire urbain. Ainsi, en décembre 1525, le contrat proposé par Jean de Paulhe, bourgeois de Gaillac, pour une métairie à Candastre, comporte une clause exigeant que « totas las galinas, aquas, pintas et autre polalhe que se noyrira a lad boria seran megieyras ", c'est-à-dire partagées à mi-fruit entre le propriétaire et le métayer, à l'exception des paons et chapons que le Gaillacois laisse dans l'exploitation et dont le croît lui appartient (Santi et Vidal $1886: 201$ ).

7 L'omniprésence des mentions de prélèvements seigneuriaux ou d'autres redevances pose nécessairement la question d'une première circulation des produits de la basse-cour, d'autant que les quantités accumulées ainsi peuvent s'avérer très importantes selon la taille des seigneuries ou des propriétés foncières. Le procès-verbal rédigé après le pillage du monastère de Schwartzentham, en Alsace, en avril 1525, impressionne par les chiffres proposés. Les pertes matérielles de la communauté religieuse sont estimées de façon exhaustive. Dans une longue liste comprenant, entre autres 64 têtes de bétail, 33 porcs, 30 pots de saindoux et 400 fromages, on note 160 poulets et pas moins de 8000 œufs tous volés ou perdus lors de l'attaque du prieuré (Bischoff 1987: 72). La quantité importante mentionnée est sans aucun doute le résultat d'un stockage après la perception des diverses redevances dues aux religieuses. Il reste qu'elle dépasse largement la consommation de l'établissement. Se pose dès lors la question d'une redistribution des denrées, voire de leur mise sur le marché.

Il ne faut toutefois pas écarter l'importance de la consommation des maisonnées seigneuriales (ou même bourgeoises). En étudiant la comptabilité de Guillaume de Murol, seigneur auvergnat du début $\mathrm{du} \mathrm{Xv}^{\mathrm{e}}$ siècle, Pierre Charbonnier a montré de façon très fine l'organisation de l'approvisionnement d'une petite cour seigneuriale. Concernant l'alimentation en viande, il apparaît très clairement que la géline est une composante essentielle du repas improvisé. L'arrivée inopinée d'invités est généralement suivie d'un repas de volailles. La raison en est bien simple : il n'y a pas d'achat nécessaire et on puise alors sur les cent vingt poules de redevances dues à Guillaume de Murol (Charbonnier 1973 : 206-207). L'importance de cet appoint alimentaire dans les maisons nobles est telle qu'on n'hésite pas à y penser au moment de rédiger son testament. Ainsi, dans le Quercy, en 1477, le vicomte Antoine de Monclar fait inscrire au notaire de Rabastens, dans son testament, qu'il laisse à sa veuve, avec d'autres biens, des revenus annuels de dix setiers d'avoine, six pipes de vin et cent poules (Marty 1908 : 217). La consommation des produits du prélèvement est également de mise dans les cours princières ou royales, comme en témoigne une quittance du 7 août 1361, produite par l'hôtel de la reine de Navarre, certifiant la réception de quatre douzaines de chapons et la même quantité de poules provenant des rentes de Conches, en Normandie, destinées à alimenter le repas des relevailles de la reine (Nortier 1971: 136). La maîtrise des dépenses des hôtels curiaux pousse les régisseurs à éviter dès qu'ils le peuvent des achats inutiles si les réserves le permettent. Le déplacement du comte de Savoie Amédée VI en Avignon, en 1362, s'accompagne du transport par voie fluviale depuis Seyssel de 231 vaches, 34 cochons, 108 
moutons ainsi que d'une multitude de poules avec le blé nécessaire à leur nourriture (Galland 1998: 56)! La part de prélèvement consacrée à la consommation nobiliaire ou princière est donc importante et touche toute la maisonnée, comme l'a montré Pierre Charbonnier avec le cas de Guillaume de Murol. On peut toutefois se demander si toutes les poules et tous les chapons reçus en redevances finissent sur les tables du seigneur ou de ses proches.

Les comptes de 1336 de la seigneurie de Bruniquel, dans le Quercy, permettent d'éclairer un autre usage des poules prélevées auprès des tenanciers. Dans le chapitre des recettes en deniers, regroupant essentiellement le paiement des différents fermages effectués pour le compte du seigneur, on note plusieurs ventes de grains et cette mention:

De VIIIXX et I [161] parelhs de galinas vendutz per Guilhem Filhol, serven, à Royre, polalhier de Tolosa, per cascu parelh XII d. tor. per tot VIII lib. XIIII s. V d. tor.

(Alauzier 1953, 365).

La vente de 161 paires de poules, soit 322 au total, à un poulailler de Toulouse témoigne de façon évidente d'une mise sur le marché du surplus des prélèvements. Le nombre d'animaux vendus est relativement élevé et sous-entend une organisation précise. Les régisseurs seigneuriaux stockent-ils provisoirement les gallinacés? Le poulailler toulousain effectue-t-il lui-même le prélèvement? Ou est-il présent le jour du paiement des coutumes? On peut également s'interroger sur le transport de plus de trois cents volailles sur une distance d'une cinquantaine de kilomètres séparant Bruniquel de la ville rose. On a vu, avec l'exemple du déplacement d'Amédée VI à Avignon en 1362, l'ampleur organisationnelle que pouvait prendre un tel déplacement (il est vrai, pour ce cas, princier). Toujours est-il que l'aire d'approvisionnement du poulailler Royre semble relativement vaste, s'appuyant certainement sur un réseau d'interconnaissances pour fournir sa part du marché toulousain. Cet achat montre en effet qu'il est essentiel de dépasser la question du prélèvement pour comprendre l'économie volaillère médiévale.

\section{Le marché des volailles et ses ramifications}

11 En étudiant, à partir de la comptabilité d'un hôpital, l'évolution des prix et des salaires à

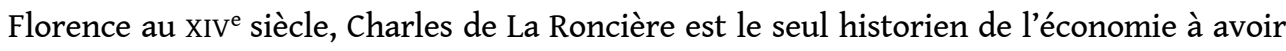
analysé précisément les conjonctures autour des produits de la basse-cour à l'époque médiévale (La Roncière 1982: 193-210). Ses conclusions proposent un regard sur la question du point de vue du marché urbain qui constitue le maillon final de la circulation de la volaille. Les séries statistiques qu'il a dressées permettent tout d'abord de replacer poulets et œufs dans la consommation urbaine. Si le prix de ces produits connait, comme pour tout le secteur alimentaire, une hausse tout au long du XIV siècle (à l'exception d'une dépression au tournant des années 1350-1360), on doit souligner sa relative régularité. Contrairement aux céréales, les crises cycliques sont rares et rapidement amorties. Étonnamment, il n'y a pas de liens entre les prix des grains et celui des produits de basse-cour et, de fait, œufs et poulets peuvent constituer des ressources nutritives stables et relativement bon marché qu'on trouve facilement dans les milieux populaires compte tenu de prix relativement bas. La cherté du grain dans la décennie précédant la peste de 1348 provoque toutefois une baisse significative de la consommation de volaille, certainement parce que le poids du pain dans le budget alimentaire est devenu trop lourd pour pouvoir acheter de la viande en sus. Après l'épidémie, et bien que les prix ne redescendent pas, le poulet reprend sa place sur la table du consommateur. Les œufs ne 
suivent pas la même logique. Leur prix plus abordable en fait un substitut à la viande lors de la crise précédant la peste. A contrario, leur part diminue dans la seconde moitié du XIV ${ }^{\mathrm{e}}$ siècle au profit de la viande, sans doute parce que le rôle de substitut de celle-ci est devenu caduc. Les prix de ces produits sont marqués par une forte saisonnalité bien évidemment liée au cycle naturel de ponte des gallinacés. Pour les œufs, les prix sont au plus bas en mai-juin, alors que la ponte vient de reprendre, et au plus fort en décembrejanvier. Entre février et avril, il n'y a pratiquement plus d'achat d'œufs, temps de carême et pénurie se recoupant de façon heureuse. Le prix des poulets suit également le cycle naturel de vie et de croissance de ces animaux. Si les prix restent stables du printemps à l'automne, ils augmentent à partir de novembre pour atteindre leur apogée en janvierfévrier. Ces grandes tendances cachent toutefois des micro-variations journalières qui reflètent les conditions d'approvisionnement. En effet, quand il est possible de suivre une même revendeuse, on s'aperçoit que les prix de vente connaissent de minimes modulations d'un jour à l'autre, liées à la nécessité de répercuter ses prix d'achat auprès des paysans. Faisant le tour des villages du contado, la revendeuse doit négocier chaque jour avec des éleveurs différents et ne parvient pas à stabiliser ses prix de revente tout en s'assurant une marge égale. Même si, compte tenu de la documentation, le marché urbain est mieux connu, c'est bien au village que tout se joue puisque c'est essentiellement là que s'organise l'élevage.

Pénétrer à l'intérieur des villages et saisir le quotidien dont relève le petit élevage n'est pas la tâche la plus évidente pour l'historien médiéviste. Les registres de justices seigneuriales ou consulaires, produits par les juridictions locales, permettent une histoire du monde rural par le bas (Wilmart 2017b). L'affaire qui anime en octobre 1353 le petit village de Mireval-Lauragais, au sud de Castelnaudary, présente des renseignements précieux pour comprendre l'aviculture domestique des campagnes du Languedoc ${ }^{3}$. Comme souvent dans ce type de registre, la querelle exposée devant les consuls de Mireval est banale: deux femmes, Genser et Rixende, se disputent au sujet d'une poule rousse (gallinam rossam). L'une des deux l'a vendue à un villageois pour deux sous trois deniers, et la seconde prétend qu'elle lui appartenait. En fait, les deux protagonistes sont cousines et voisines. Pour optimiser leurs espaces et leurs activités, elles ont placé leurs poules dans un poulailler commun. Pour reconnaitre leurs volailles, une marque est apposée à chaque bête appartenant à Genser qui leur enlève trois ongles à la patte droite, évitant, normalement, tout risque de confusion. Les différents témoignages recueillis permettent d'ajouter des précisions sur l'organisation de la petite affaire. Les deux femmes distinguent clairement des poules couveuses (pro fovendo ovem) des autres, sans doute cantonnées à la ponte car plus jeunes. De plus, leur commerce ne se limite pas au village puisqu'elles vont régulièrement en vendre à Fanjeaux, à une dizaine de kilomètres de distance. Détail presque ethnographique, le mari de la femme qui a vendu la poule précise qu'il voit très bien de quelle poule il s'agit puisqu'elle venait régulièrement manger dans sa maison. De cette précision, on doit déduire que si le poulailler est commun, les gallinacés jouissent toutefois d'un régime de semi-liberté leur permettant de paraitre véritablement familiers à leurs propriétaires. La vente qui a provoqué la querelle se déroule sans marché établi. L'acheteur explique que, ayant vu que Rixende était dans son verger, il s'est adressé à sa cousine occupée à sarcler son jardin à proximité de sa maison. Cette dernière l'a alors envoyé au poulailler chercher deux poules et, quand il est revenu, elle a vérifié à l'aide de la marque si l'une était à elle, l'a choisie et a relâché l'autre. On doit ici relever deux éléments : d'une part, tous les villageois ne sont pas 
propriétaires d'un poulailler, d'autre part, l'acheteur sait très bien à qui il s'adresse, ce qui signifie qu'on sait dans le village qui a des poules et qui les vend. Cette affaire permet bien d'articuler l'ensemble des rouages de l'économie volaillère. Tout d'abord, la tenue d'un poulailler est un investissement et il faut parfois se regrouper pour en avoir un qui soit suffisamment productif. Ensuite, il existe bien une technique avicole minimale : on achète des poules (plus vieilles?) destinées à couver, puis on les revend. Enfin, il existe bien deux marchés: l'un villageois, basé sur un réseau de voisinage qui échappe à l'historien s'il ne dispose pas de ce type de sources, et l'autre urbain, dans lequel les villageoises de Mireval semblent faire de la vente directe, même s'il n'est pas impossible qu'elles revendent leur production à un volailler.

13 Du commerce entre villageois, on doit sans doute déduire que, même si la coutume impose une redevance en poules, tous n'ont pas de volailles à domicile. L'image d'un petit élevage domestique dans chaque maison, qui avait pu être forgée par cette généralisation de ce type d'obligation, est à relativiser. Ces mêmes coutumes ou statuts se font d'ailleurs parfois l'écho d'un recensement (certainement a minima) des foyers élevant des gallinacés. Ainsi, à Polastron, en Gascogne, les coutumes de la fin du XIII ${ }^{\mathrm{e}}$ siècle précisent le déroulement d'achat seigneurial de volailles (Bladé 1864 : 70-71). En cas de nécessité liée à la présence d'hôtes ou de l'organisation d'une fête solennelle, les seigneurs peuvent venir acheter aux villageois de la volaille. Ils doivent pour cela envoyer un messager aux consuls du lieu qui l'accompagnent alors chez ceux susceptibles de fournir les bêtes nécessaires. Si l'article est conclu par une obligation de vente in fine, il témoigne surtout d'une connaissance par les autorités villageoises du cheptel disponible. Ces règlements protègent également la source de revenus complémentaires que permet l'entretien d'un poulailler en limitant les prélèvements arbitraires destinés à alimenter la table seigneuriale. Au Fossat, dans le comté de Foix, les coutumes accordées en 1274 stipulent sans ambiguïté l'interdiction faite au seigneur et à ses familiers de prendre autoritairement les poules des villageois, sous peine d'être banni du lieu (Pasquier 1897 : 294-295, article 9).

La reconnaissance du poids économique du poulailler prend une nouvelle dimension au cours des XIV et $\mathrm{XV}^{\mathrm{e}}$ siècles quand, en Languedoc, les estimes, destinées à définir l'assiette de l'impôt selon les biens de chacun, prennent de plus en plus en compte les biens meubles (Rigaudière 1989). Dans un premier temps évalués globalement, ils sont l'objet d'une attention particulière au cours du $\mathrm{Xv}^{\mathrm{e}}$ siècle. Toutefois, si le cheptel est désormais bien comptabilisé, l'évaluation du petit bétail (porcs et volailles) reste l'exception. À Castres, l'ordonnance consulaire de 1383 fixant les modalités de recensement des biens inclut tous les animaux, y compris les volailles. En 1464, certaines paroisses du Vivarais recensent volailles et lapins dans les biens meubles listés dans les estimes (Rigaudière $1989: 67)^{4}$.

Même si les poules ne sont pas des animaux imposants, les autorités villageoises règlementent parfois leur circulation, pour éviter une trop grande promiscuité dans l'espace public, voire pour éviter qu'un élevage trop important en accapare une partie. En Île-de-France, à Charny, près de Meaux, la justice seigneuriale intervient ainsi en août 1478 pour interdire la présence de "pourceaulx, cochons, poulles et aultres volailles » sur les tas de paille laissés dans la rue devant les maisons, sous peine de soixante sous d'amende (Registres 2000 : 188). En Languedoc, à Villeneuve-lès-Béziers, les statuts défendent d'installer des "gallinas et pollets dedins las cavas comunas ", c'est-àdire dans les fossés entourant le village, sous peine de cinq sous d'amende et de la 
confiscation de la volaille (Bédard 1927 : 201, article LXI). Une interdiction aussi précise témoigne certainement d'une tentative d'utiliser l'espace commun des fossés pour y installer un élevage plus conséquent et pallier ainsi au manque de place à proximité des maisons. Le marché étant relativement prospère, il doit exister une tentation de donner à l'élevage avicole des dimensions plus importantes, comme en témoigne en 1405 la mésaventure des religieux de l'église Santa Maria Nova de Rome. Ayant hérité la moitié d'un domaine agricole, ils louent leurs biens au propriétaire de l'autre moitié qui va s'avérer mauvais payeur. Au cours de la procédure qui est lancée contre lui, on apprend que celui-ci a installé dans les bâtiments un élevage de volailles (poules et canards) qui prend un tel essor que les moines ne peuvent plus pénétrer à l'intérieur quand ils décident de le récupérer (Maire-Vigueur 1974 : 125).

L'élevage touche également le milieu urbain, sans qu'on puisse faire de lien avec une éventuelle spéculation sur le marché de la volaille. Ainsi, à la fin du XIve siècle, à Avignon, une des maisons qui appartient au marchand cordier Jean Teisseire, et qui semble servir d'entrepôt, abrite une trentaine de poules (Hayez 1996: 445). À Paris, on rencontre parfois des poulaillers près des maisons. Ainsi, un censier de 1461 décrit l'hôtel du Chapeau-Rouge, rue Saint-Antoine, et signale qu'il y a dans sa cour « un puiz mitoien et un gelinier et chambres aisiées» (Mirot 1911: 85). Toutefois, les cas de poulaillers parisiens restent rares, certains propriétaires n'hésitant pas à laisser leurs poules s'ébattre dans l'étable ou dans la cour, faute de structures plus adaptées. À Paris, la volaille reste un cheptel d'appoint : seuls $7 \%$ de ceux qui en possèdent n'ont pas d'autres animaux. Au contraire, près de $80 \%$ de ceux qui ont des bêtes chez eux n'ont pas de volailles. Même si certains élevages peuvent aller jusqu'à quarante poules, la moyenne reste bien plus basse. Un tiers des propriétaires en ont moins de dix, les deux tiers moins de vingt (Gurvil 2010 : 393-394). Le commerce des volailles ne peut donc pas être assuré par une aviculture urbaine. L'approvisionnement vient bien de l'extérieur et est fortement règlementé.

Si les activités marchandes autour des volailles sont attestées dans toutes les villes, notamment par la présence dans la plupart d'une rue dite de la poulaillerie, les sources écrites restent moins prolixes pour en appréhender le fonctionnement. Les statuts de métiers édictés pour les volaillers ou les rôtisseurs permettent une première approche. Ces règlements font de ces professionnels les acteurs les plus visibles d'un commerce dont ils ne sont en fait qu'un maillon. Un des objectifs de la règlementation urbaine est d'organiser l'approvisionnement de toute la population. Pour assurer la qualité des produits, le marché volailler est, à l'instar de la boucherie, étroitement surveillé. Ainsi, à Lille, au $\mathrm{Xv}^{\mathrm{e}}$ siècle, la municipalité rémunère des inspecteurs de la volaille, chargés de vérifier la marchandise (Pétillon 1983 : 424). Les statuts, en encadrant les activités des métiers qu'ils règlementent, permettent également d'apercevoir l'organisation de l'approvisionnement. À Paris, ceux des volaillers sont connus dès la fin du XIII ${ }^{\mathrm{e}}$ siècle par le recueil d'Étienne Boileau, puis par une série d'ordonnances entre les XIV et $\mathrm{XVI}^{\mathrm{e}}$ siècles (Lespinasse $1886: 342-350$ ). Tous ces textes s'accordent sur un point : l'interdiction faite aux professionnels d'acheter des volailles sur le marché (auquel ils sont par ailleurs obligés de se rendre) avant une certaine heure, midi aux XIII ${ }^{e}$ et XIV ${ }^{e}$ siècles, onze heures au $\mathrm{XV}^{\mathrm{e}}$ siècle. Cette contrainte n'est pas propre aux grandes villes puisqu'on la retrouve dans les statuts des cuisiniers et rôtisseurs de Bayeux. Là, le texte de 1472 fixe à dix heures le début de l'ouverture du marché aux professionnels, précisant «afin que les bourgeois de ceste dicte ville en puissent estre fournis » (Le Paulmier 1896 : 10). Le circuit 
de commercialisation peut donc être décrit grâce à ces interdictions et obligations. Les paysans, ayant élevé eux-mêmes des poules ou les ayant achetées à leurs voisins, se rendent en ville et s'installent sur les emplacements indiqués par les règlements urbains. Là, leur marchandise est contrôlée : les volailles doivent être vivantes et en bonne santé, seuls les oiseaux sauvages et le petit gibier pouvant être vendus morts. L'achat est alors pendant une grande partie de la matinée réservé aux particuliers qui peuvent ainsi s'approvisionner en viande blanche et œufs. Puis, à l'heure prévue, les volaillers et les rôtisseurs, auxquels on doit ajouter les aubergistes et les taverniers, viennent acheter les produits qu'ils pourront à leur tour revendre, tels quels ou préparés, à leur clientèle. Commence alors un second cycle de commercialisation aux prix plus élevés pour assurer aux revendeurs leurs marges.

On le voit, la commercialisation des gallinacés, si elle semble facile dans les campagnes, fait l'objet d'un encadrement très strict en milieu urbain. Comme le montre l'exemple florentin, la consommation de volailles et de leurs produits reste importante tout au long des derniers siècles du Moyen Âge, à quelques périodes d'exception près. Le marché dépend alors étroitement du monde paysan : c'est lui qui livre les places commerçantes, qui fixe les prix, qui vend directement au plus grand nombre. Les professionnels de la revente sont servis en dernier et ne sont pas libres de s'approvisionner où bon leur semble. Cette mécanique semble toutefois s'enrayer à l'époque moderne. Au milieu du $\mathrm{XVII}^{\mathrm{e}}$ siècle, les tentatives royales de créer des offices de vendeurs de volailles se soldent par des échecs successifs, alors même que la corporation des volaillers éprouve les pires difficultés à se maintenir, notamment à cause de la concurrence accrue des rôtisseurs (Lespinasse 1886 : 349-351). On préfère dès lors sans doute acheter plus cher une volaille cuite, plutôt que vivante. Mais ce changement n'est pas qu'une affaire de goût. Dès la fin du $\mathrm{XVI}^{\mathrm{e}}$ siècle, des voix s'élèvent pour imaginer une nouvelle économie volaillère.

\section{Vers un nouveau modèle?}

En 1569 paraît un petit traité rédigé par Prudent Le Choyselat, intitulé Discours oeconomique, non moins utile que récréatif, montrant comme de $500 \mathrm{l}$. pour une fois employées, l'on peult tirer par an $4500 \mathrm{l}$. de proffict honeste. Sa réédition en 1572 voit son titre modifié, précisant que le plan proposé se fait « par le mesnagement de poulles ». Souvent cité dans son édition de 1612, car plus facile d'accès, c'est près d'un demi-siècle avant cette date que l'on doit situer le contexte de sa rédaction ${ }^{5}$. En effet, pour en comprendre l'originalité, il faut d'abord définir sa place dans les discours agronomiques. Sa publication suit de quelques années celle de l'Agriculture et Maison rustique de Charles Estienne et Jean Liébault (1564), qui est elle-même la traduction remaniée du Praedium Rusticum publié par le premier en 1554. Le Théâtre d'agriculture d'Olivier de Serres est, quant à lui, bien plus tardif puisqu'il paraît en 1605. Il y a sans aucun doute un projet éditorial dans lequel s'inscrit Prudent Le Choyselat et qui suit le succès de la Maison rustique. L'imprimeur du Discours oeconomique ne s'arrête en effet pas là. En 1571, il propose une traduction française des Secrets de la vraie agriculture de l'auteur italien Augustin Gallo. Le traducteur, François de Belleforest, écrivain gascon qui mène une carrière très active à Paris (Simonin 1992), rédige d'ailleurs en 1569 un éloge en soixantesix vers du traité de Le Choyselat.

Prudent Le Choyselat (mort en 1577) n'est ni naturaliste, ni agronome, mais procureur à Sézanne, en Brie champenoise. Sa famille est incontournable dans cette petite ville où elle 
occupe nombre d'offices (avocat, notaire, procureur). En 1560-1561, c'est lui qui représente la communauté aux États généraux d'Orléans où il se lie avec Jacques de Silly, comte de Rochefort et damoiseau de Commercy (et entre autres seigneur de Montmirail, près de Sézanne), l'orateur de la noblesse qui s'en prend avec virulence au pouvoir temporel de l'Église. Prudent Le Choyselat lui dédie d'ailleurs son traité. De fait, son texte s'inscrit bien dans son époque marquée par les guerres de Religion et les troubles de 1567 qu'il cite dès les premières pages. C'est à la fois un discours moral, contre l'oisiveté, conseillant de placer son argent sans usure, et un discours économique comme le montre la lettre d'introduction adressée à un ami gentilhomme qui aurait tout perdu pendant les guerres. Il s'agit dès lors de reconstruire une économie sur de nouvelles bases. La brièveté du Discours vise avant tout à l'efficacité. Bien sûr, Prudent Le Choyselat y montre l'étendue de son savoir livresque en citant les auteurs antiques (Columelle, Varon, Aristote, etc.) et médiévaux (Pierre de Crescens) mais aussi ses contemporains que sont Charles Estienne, Pierre Belon, François Rabelais, Guillaume Budé ou encore Érasme. Cependant, il se détache de ces autorités à la fois par l'originalité de son projet et par des éléments empiriques qu'il introduit çà et là.

Dans sa lettre introductive, Prudent Le Choyselat s'attache à définir l'économie traditionnelle des grosses exploitations agricoles qui se sont développées au cours du Moyen Âge :

Ton revenu véritablement consistoit en mesnage de toutes sortes de bestial, bœufs, vaches, jumens, brebis, moutons et volailles. (Le Choyselat 1581 : f. 6)

Il propose alors d'opérer trois changements majeurs. Le premier est un véritable renversement de l'économie rustique puisqu'il incite à se concentrer sur un seul type d'élevage, ici l'aviculture. La deuxième nouveauté est la taille de l'élevage envisagé puisque que les trois cents livres de départ doivent être investies dans l'achat de 1200 poules. Le troisième changement se fait dans le choix du lieu de l'exploitation. Désormais, celui-ci doit se baser non pas sur un héritage patrimonial, mais sur la proximité d'un marché, d'un débouché idéal pour la production envisagée.

23 La première étape consiste donc à choisir aux environs de la ville de Paris « quelque lieu bien basti de manoir et estables, qui ayt court et accin de la continance de deux arpens, plus ou moins, clos et fermé de murailles assez hautes, avec quelque deux autres arpens ès environs du clos» (Le Choyselat 1581: f. 6v-7), soit une petite exploitation de 1,2 hectare à prendre en ferme pour en réduire le coût. Les étables déjà existantes sont alors à transformer en "gélinier ", pratique que nous avons pu observer avec l'affaire des religieux de Santa Maria Nova de Rome. Pour cela, il faut y créer un étage terrassé d'argile et y dresser des juchoirs. En dessous, on doit placer des paniers amples garnis de foin qui, pour l'auteur, est plus doux que la paille et moins touché par les poux et les vermines. À proximité, il est nécessaire de prévoir un demi-arpent de terre labourée pour que les poules puissent s'y promener et gratter la terre.

24 Une fois les lieux d'exploitation en place, il faut acquérir les animaux qui vont l'occuper. Prudent Le Choyselat déconseille fortement les dindes qui sont de «vrais greniers à avoine » et privilégie les poules des pays d'Anjou, de Tournon et du Lodunois car ce sont les pays les moins touchés par les guerres. Prudent Le Choyselat ne s'attarde donc pas sur des races, dont l'élaboration se fera plus tard ${ }^{6}$, mais lie des aires géographiques à des contextes de développement économique, de nutrition et d'état sanitaire résultant des évènements récents. Quant aux critères de sélection des individus, le procureur de Sézanne se contente de reprendre les prescriptions des auteurs antiques. Se basant sur le 
prix de cinq sous fixé par ordonnance royale en 1567, il estime la dépense à 300 livres auxquelles s'ajoute l'achat d'un coq pour dix poules, soit 42 livres pour 120 coqs à 7 sous.

Après ces considérations de départ, Prudent Le Choyselat envisage l'organisation de l'élevage proprement dit. Celui-ci nécessite l'emploi de quatre servantes pour s'occuper des poules dont la vie est réglée. Le poulailler est ouvert le matin pour les faire sortir et refermé à $15 \mathrm{~h}$ en hiver et à $17 \mathrm{~h}$ en été. Après la sortie des animaux, les servantes s'attèlent au nettoyage du poulailler, des juchoirs et des abreuvoirs, au ramassage des œufs, à la distribution de la nourriture (orge, avoine, vesces). Pour éviter toute épidémie et prévenir des parasites, le foin des paniers est changé une fois par semaine.

La grande nouveauté proposée par Prudent Le Choyselat consiste en la maîtrise totale du circuit de commercialisation des œufs. C'est en effet de ces produits que les revenus doivent être tirés et non de la vente de volaille. L'objectif est de vendre la production quotidienne en œufs frais des 1200 poules, à un prix plus élevé que les autres œufs vendus habituellement en argumentant sur leur fraîcheur. Selon une logique commerciale d'une modernité confondante, il s'agit de présenter un produit frais comme un produit de luxe. Pour cela, l'auteur préconise d'approcher douze ou quinze médecins répartis dans tous les quartiers de Paris et de les convaincre de conseiller à leurs malades la consommation d'œufs frais :

$\mathrm{Tu}$ leur feras entendre que par chacun jour tu as moyen de fournir leurs patiens d'œufs frais pondus du jour au lendemain, dont ils les pourront asseurer. Tu les advertiras aussi des revenderesses que tu y commettras pour la distribution. (Le Choyselat 1581 : f. 13v).

L'appui des médecins, qui jouent ici un rôle à la fois de publicité et de rabattage de la clientèle, est renforcé par la mise en place d'un réseau de revendeuses ou regrattières chargées de vendre les œufs frais en s'engageant à de ne pas en proposer d'autres, garantissant ainsi une exclusivité du produit. Elles aussi sont réparties dans différents secteurs de Paris. Entre l'exploitation et le marché parisien, un transport quotidien est organisé par deux ânes partant chaque matin de la ferme. On voit donc que Prudent Le Choyselat envisage un circuit de distribution particulièrement bien huilé, avec des arguments commerciaux et un marché géographiquement et socialement quadrillé.

L'auteur se lance ensuite dans un calcul précis du profit, sur lequel nous ne nous attarderons pas. Certains ont voulu y voir le premier plan d'affaires (Marco et Noumen 2015), mais la question de la rationalité de l'économie rurale n'est pas nouvelle. Le seul revenu est la vente de l'œuf, vendu six deniers pièce, ce qui est cher mais, compte tenu de la population parisienne, Prudent Le Choyselat fait le pari qu'ils trouveront preneur. Il propose deux scénarios de rentabilité, l'un avec une production de 800 œufs par jour, l'autre avec 600, et le bénéfice calculé se trouve alors entre 3447 et 7300 livres par an. Les dépenses envisagées portent sur la nourriture des volailles et des ânes, le loyer du manoir, le salaire des quatre servantes, de l'ânier et des vendeuses, la rétribution des médecins. Outre quelques erreurs de calcul, on doit noter que les dépenses sont simplifiées, notamment pour les salaires versés qui sont équivalents entre hommes et femmes ce qui ne correspond pas à la réalité telle que nous la connaissons. De plus, Prudent Le Choyselat ne prend en compte ni le renouvellement d'une partie des poules, ni l'autoproduction de la nourriture des volailles. Ces quelques erreurs ne retirent rien à la nouveauté de son Discours oeconomique, même si l'auteur affirme dès le titre qu'il est « non moins utile que récréatif». On ne peut donc éluder le côté ludique du traité de Le Choyselat, mais il faut souligner que c'est un véritable succès d'édition (Huzard 1830). Il 
est réédité en 1569, 1572, 1575, puis à titre posthume en 1581, 1585 (distribué par les colporteurs), 1586 (à Lyon), 1595, 1598 (à Fréjus) et 1612 (à Rouen). Le succès du Théâtre d'agriculture d'Olivier de Serres vient interrompre la vague de rééditions. Ajoutons que le traité est traduit en anglais en 1577, puis de nouveau imprimé dans cette langue en 1580, et connaît une édition allemande en 1615.

Ce succès doit être toutefois confronté à un échec: il ne semble pas que les idées de Prudent Le Choyselat se diffusent. Il y a bien sûr quelques héritiers, apparemment involontaires, à l'instar de Vauban qui, dans son recueil d'Oisivetés ou ramas de plusieurs sujets à ma façon, fait un calcul similaire mais avec des porcs (Virol 2007 : 627-637). Encore au XVIII ${ }^{\mathrm{e}}$ siècle, Réaumur tente de proposer une rationalisation de l'aviculture avec son Art de faire éclore et élever en toute saison des oiseaux domestiques. Mais on ne trouve en réalité aucune trace d'application concrète. Les inventaires de fermes étudiés par JeanMarc Moriceau pour l'île-de-France ne montrent aucune exploitation avicole spécialisée (Moriceau 1994). En fait, le système de Le Choyselat se heurte à la police des corporations dont il n'a pas tenu compte. L'exemple de l'évolution des statuts des cuisiniers et rôtisseurs de la ville de Bayeux illustre parfaitement ce problème. Lors de leur refonte en 1735, les statuts intègrent un article dénonçant l'accaparement par des particuliers des colombiers des campagnes alentour (Le Paulmier 1896 : 22). Certains individus « arrêtent ou louent des coulombiers ou fuies à l'année [...] et par ce moyen empêchent que les fermiers ou propriétaires n'apportent leurs pigeons aux marchés de cette ville». Montrant du doigt les regrattiers, tentés de maîtriser toute la chaîne de la production à la distribution, les rôtisseurs s'arrogent le droit d'intervenir, de saisir les marchandises et de mettre à l'amende les accapareurs. Si cet article a été rajouté au XVIII ${ }^{\mathrm{e}}$ siècle, c'est bien qu'il a dû exister des tentatives de modification de l'économie volaillère dont le traité de Prudent Le Choyselat marque les prémices. En louant les pigeonniers, les regrattiers sont à la fois producteurs et vendeurs, maîtrisent les prix et les bénéfices. Mais ils se heurtent alors à des corporations encore puissantes. Grâce à elles, l'économie volaillère médiévale, telle que décrite dans cette article, semble se prolonger pendant l'époque moderne. Il reste qu'une recherche plus poussée en archives sur la longue durée permettrait sans doute d'affiner les évolutions de l'aviculture au cours des XVII ${ }^{\mathrm{e}}$ et XVIII ${ }^{\mathrm{e}}$ siècles.

\section{BIBLIOGRAPHIE}

Alauzier L. d' 1953 - Un compte du XIV e siècle de la vicomté de Bruniquel. Bulletin philologique et historique jusqu'à 1715, années 1951-1952 : 357-376.

Bédard P.-J. 1927 - Les statuts de Villeneuve-lès-Béziers en 1513. Bulletin de la Société archéologique, scientifique et littéraire de Béziers 45 : 169-206.

Bischoff G. 1987 - Huit mille œufs et cinq cents livres. Le pillage du couvent de Schwartzentham en avril 1525 d'après la relation de la prieure Ursula Vorburger. Archives de l'Église d'Alsace 66 : 63-73.

Bladé J.-F. 1864 - Coutumes municipales du département du Gers. Paris, Durand, 255 p. 
Charbonnier P. 1973 - Guillaume de Murol. Un petit seigneur auvergnat au début du XV siècle. Clermont-Ferrand, Institut d'études du Massif Central, $527 \mathrm{p}$.

Charbonnier P. 1980 - Une autre France. La seigneurie rurale en Basse Auvergne du XIV au XVI siècle. Clermont-Ferrand, Institut d'études du Massif Central, $1294 \mathrm{p}$.

Crescens P. de 1516 - Le livre des prouffitz champestres et ruraux. Paris.

Feller L. (Ed.) 2009 - Calculs et rationalités dans la seigneurie médiévale : les conversions de redevances entre $\mathrm{XI}^{e}$ et $\mathrm{XV}^{e}$ siècles. Paris, Publications de la Sorbonne, $365 \mathrm{p}$.

Fillet L. 1890 - État des revenus de l'évêché de Die vers 1474, suivi de celui des chapellenies fondées dans les églises Notre-Dame de Die et Saint-Sauveur à la même époque. Bulletin du Comité des travaux historiques et scientifiques, section de d'histoire et philologie : 23-37.

Fourquin G. 1964 - Les campagnes de la région parisienne à la fin du Moyen Âge, de la fin $d u X X^{e}{ }^{e} a u$ début du XVI siècle. Paris, PUF, 589 p.

Galland B. 1998 - Les papes d'Avignon et la Maison de Savoie (1309-1409). Rome, Publications de l'École française de Rome, 516 p.

Gurvil C. 2010 - Les paysans de Paris du milieu du XVee au début du XVII siècle. Paris, Honoré Champion, $694 \mathrm{p}$.

Hayez A.-M. 1996 - Le patrimoine urbain d'un marchand cordier avignonnais : Jean Teisseire († 1384). Bibliothèque de l'École des chartes 154 (2) : 427-484.

Huzard J.-B. 1830 - Notice analytique et bibliographique de l'ouvrage de Prudent Le Choyselat sur les avantages que l'on peut retirer des poules. Paris, $24 \mathrm{p}$.

Laporte P. 1911 - Coutumes d'Endoufielle (XIII e siècle). Auch, Cocharaux, 21 p.

La Roncière C. de 1982 - Prix et salaires à Florence au XIV siècle. Rome, Publications de l'École française de Rome, 888 p.

Le Choyselat P. 1581 - Discours oeconomique non moins utile que récréatif, montrant comment par le mesnagement de poulles de cinq cents livres pour une fois employées on peult tirer par an quatre mille cinq cent livres de proffict honneste. Paris, Nicolas Chesneau, $26 \mathrm{f}$.

Le Paulmier J. 1896 - Les statuts des maîtres cuisiniers de la ville de Bayeux, 1473-1731. Bayeux, Duvant, $28 \mathrm{p}$.

Lespinasse R. de 1886 - Les métiers et corporations de la ville de Paris : XIV -XVIII siècle, t. I, Les métiers de l'alimentation. Paris, Imprimerie nationale, $711 \mathrm{p}$.

Maire-Vigueur J.-M. 1974 - Les casali des églises romaines à la fin du Moyen Âge (1348-1428). Mélanges de l'École française de Rome 86 (1) : 63-136.

Marco L. \& Noumen R. 2015 - The First Business Plan in France of Prudent Le Choyselat, 1569-1612. Saint-Denis, Édi-Gestion, 126 p.

Marandet M.-C. 2006 - Les campagnes du Lauragais à la fin du Moyen Âge. Perpignan, Presses universitaires de Perpignan, $464 \mathrm{p}$.

Marty E. 1908 - Archives des notaires de Rabastens (suite). Revue historique, scientifique et littéraire du département du Tarn $25: 202-220$.

Mirot L. 1911 - Les origines de l'hôtel de Sully et la censive du prieuré de la Couture-SainteCatherine dans la rue Saint-Antoine. Bulletin de la Société de l'histoire de Paris et de l'île-de-France 38 : 77-95. 
Moriceau J.-M. 1994 - Les fermiers de l'Île-de-France. L'ascension d'un patronat agricole (XV'-XVIII siècles). Paris, Fayard, 1070 p.

Nortier M. 1971 - Les sources de l'histoire de la Normandie à la Bibliothèque nationale de Paris. Le fonds français des manuscrits, t. II, Quittances administratives, règne de Jean II le Bon (suite). Annales de Normandie 21 : 133-148.

Oschinsky D. (Ed.) 1971 - Walter of Henley and other treatises on estate management and accounting. Oxford, Clarendon Press, 504 p.

Parfouru P. 1900 - Compte du temporel de l'évêché de Meaux (1425-1426). Paris, Picard, 48 p.

Pasquier F. 1897 - Coutumes du Fossat dans le comté de Foix d'après une charte de 1274. Annales du Midi 9 : 257-322.

Pétillon C. 1983 - Le personnel urbain de Lille (1384-1419). Revue du Nord 65 (257) : 411-427.

Registres 2000 - Registres des justices de Choisy-le-Temple et Châtenay, 1448-1478. Édition des registres Z $Z^{2}$ 761 et 902 des Archives nationales. Paris, Honoré Champion, 288 p.

Rigaudière A. 1989 - Connaissance, composition et estimation du moble à travers quelques livres d'estimes du Midi français ( $\mathrm{XIV}^{\mathrm{e}}-\mathrm{XV}^{\mathrm{e}}$ siècles). In : Les cadastres anciens des villes et leur traitement par l'informatique. Actes de la table ronde de Saint-Cloud, 31 janvier-2 février 1985. Rome, Publications de l'École Française de Rome : 41-81.

Sahuc J. 1895 - Saint-Pons-de-Thomières. Inventaire sommaire des archives communales antérieures à 1790. Montpellier, Ricard, $151 \mathrm{p}$.

Sahuc J. 1903 - Charte des libertés et franchises accordées aux habitants de la ville et de la seigneurie d'Olargues en 1289. Mémoires de la Société archéologique de Montpellier 3 : 295-317.

Santi L. de \& Vidal A. 1886 - Deux livres de raisons : 1517-1550 avec des notes et une introduction sur les conditions agricoles et commerciales de l'Albigeois au XVI siècle. Paris, Champion, 689 p.

Sherwood M. 1941 - Un registre de la cour criminelle de Mireval-Lauragais au XIV ${ }^{\mathrm{e}}$ siècle. Annales du Midi 53 (210) : 169-182.

Simonin M. 1992 - Vivre de sa plume au XVI $I^{e}$ siècle ou la carrière de François de Belleforest. Genève, Droz, $328 \mathrm{p}$.

Tardif G. 1492 - C'est le livre de l'art de faulconnerie et des chiens de chasse. Paris.

Valladier-Chante R. 1993 - Vallon-Pont-d'Arc à la fin du Moyen Âge, une communauté paysanne du Vivarais. Valence, Éditions de la Bouquinerie, 236 p.

Virol M. (Ed.) 2007 - Les oisivetés de Monsieur de Vauban. Seyssel, Champ Vallon, 1722 p.

Wilmart M. à paraître - Les remèdes pour animaux dans l'Occident médiéval $\left(\mathrm{XII}^{\mathrm{e}}-\mathrm{XV}^{\mathrm{e}} \mathrm{s}\right.$.). In : Rieder P. et Zanetti F. (Ed.) Médicament(s), XII ${ }^{e}-\mathrm{XVIII}{ }^{e}$ siècle. Actes du colloque de Paris, 21-22 octobre 2011. Genève, Droz.

Wilmart M. 2017a - Saignées et autres manipulations vétérinaires d'après les traités d'hippiatrie et d'économie rurale de l'Occident médiéval ( $\mathrm{XII}^{\mathrm{e}}-\mathrm{XV}^{\mathrm{e}}$ siècle). In : Lorans $\mathrm{E}$. (Ed.) Le cheval au Moyen Âge. Tours, Presses universitaires François Rabelais : 65-75.

Wilmart M. 2017b - Travailler pour les autres dans un village de la région de Meaux à la fin du XV e siècle. Histoire et Sociétés rurales 47 : 7-40. 


\section{NOTES}

1. Le même auteur se prononce pour un élevage généralisé, y compris chez les plus pauvres des campagnes (Fourquin 1964 : 525).

2. Sur les conversions de redevances voir Feller (2009).

3. L'affaire est présentée dans Sherwood (1941: 179-182). Nous avons vérifié le récit sur le manuscrit original, Arch. dép. de l'Aude : 4 E 234 S 7.

4. Il y aurait une étude à effectuer sur les effectifs de poules dans les paroisses qui ont poussé le zèle fiscal jusqu'à comptabiliser les volailles, mais nous n'avons pu, faute de temps, le faire pour cet article.

5. Sur les différentes éditions du traité de Prudent Le Choyselat, voir Huzard (1830). Sur l'auteur, Marco et Noumen (2015). Nous avons, pour cet article, utilisé l'édition de 1581.

6. Voir l'article de B. Denis dans ce numéro.

\section{RÉSUMÉS}

L'élevage de poules a très peu intéressé les historiens de l'économie rurale médiévale. L'article se propose tout d'abord de relire la question des prélèvements seigneuriaux de volailles souvent cités mais peu étudiés. Se posent alors deux interrogations : la généralisation de l'élevage avicole chez les paysans et les débouchés des prélèvements (consommation nobiliaire et commercialisation). À partir de deux exemples (Florence et les campagnes du Languedoc), l'article montre l'organisation de cette commercialisation. À la fin du XVI ${ }^{\mathrm{e}}$ siècle, le modèle médiéval est remis en cause. Avec le traité de Prudent Le Choyselat (1569), on assiste à une première tentative de rationalisation économique par le développement d'un élevage intensif et spécialisé.

The poultry farming little interested the historians of the medieval rural economy. The article reconsiders at first the question of the seigniorial levies of poultry, often quoted but little studied. Then two questioning could be asked: the generalization of the poultry breeding at the peasants and the outlets of the levies (nobiliary consumption or market). From two examples (Firenze and Languedoc), the article explains the organization of this market. At the end of the 16th century, medieval model is questioned. With the treaty of Prudent Le Choyselat, we attend a first attempt of economic rationalization by the development of an intensive and specialized breeding.

\section{INDEX}

Mots-clés : élevage, économie rurale, commercialisation

Index géographique : Florence, Languedoc, France

Keywords : breeding, rural economy, commercialization, Languedoc, France, Firenze 
AUTEUR

MICKAËL WILMART

Ingénieur d'études, EHESS, CRH/GAM 